\title{
Continuous Chemical Supplement, a Novel Solution to Improve the Efficiency of Peroxide Bleaching
}

\author{
Xianqi Sun ${ }^{1 a}$, Haizhen Shi² ${ }^{2 b}$ Jianfeng Jic ${ }^{3 c}$, Lichun Zhou ${ }^{3 d}$ \\ ${ }^{1}$ Tianjin University of Science \& Technology, Tianjin 300457, China \\ ${ }^{2}$ Longyou Speciality Paper Research Institute, Longyou 324400, Zhejiang Province, China \\ ${ }^{3}$ China National Pulp and Paper Research Institute, Beijing 100102, China \\ asxq7310@126.com, bshzqzfy@126.com, ca07130410@163.com, d7248344090@163.com
}

Keywords: Continuous chemical supplement; Bleaching efficiency; peroxide decomposition; Poplar chemi-mechanical pulp

Abstract: In conventional peroxide bleaching of chemi-thermomechanical pulp (CTMP), the bleaching efficiency of hydrogen peroxide $\left(\mathrm{H}_{2} \mathrm{O}_{2}\right)$ is far lower than its expected level. In this paper, a novel bleaching method is proposed to improve the $\mathrm{H}_{2} \mathrm{O}_{2}$ bleaching efficiency of poplar CTMP. This novel method, namely Continuous Chemical Supplement (CCS), maintained constant concentrations of $\mathrm{H}_{2} \mathrm{O}_{2}$ and total alkali in the bleaching system for a long time by supplementing continuously $\mathrm{H}_{2} \mathrm{O}_{2}$ and $\mathrm{NaOH}$ solutions using peristaltic pumps. The $\mathrm{H}_{2} \mathrm{O}_{2}$ decomposition was reduced and the brightness was improved. Consequently, the bleaching efficiency was improved. The experimental results indicated that the bleaching efficiency reached 16.5 by CCS, which was significantly higher than that obtained by the traditional high-consistency bleaching method (7.4). This novel bleaching method may be helpful to industrial production practice.

\section{Introduction}

The bleached chemi-mechanical pulp is commonly used in various grades of paper products such as lightweight coated paper and white cardboard [1-3]. Since 1990's, chemi-mechanical pulping has been rapidly developing. Hydrogen peroxide $\left(\mathrm{H}_{2} \mathrm{O}_{2}\right)$, as an environmentally-friendly bleaching agent, is widely used in the both pulping process [4]. However, the bleaching efficiency, which is defined as a pulp brightness gain per unit of $\mathrm{H}_{2} \mathrm{O}_{2}$ consumption, is far lower than the expected level [5-7]. This mainly resulted from the undesired decomposition reaction of $\mathrm{H}_{2} \mathrm{O}_{2}$ [8]. Therefore, one of effective approaches to improve the bleaching efficiency is inhibiting $\mathrm{H}_{2} \mathrm{O}_{2}$ from undesired decomposition.

The chemical concentrations (mainly $\mathrm{H}_{2} \mathrm{O}_{2}$ and total alkali) in a peroxide bleaching system have an important influence on the bleaching efficiency. High chemical concentrations can accelerate bleaching reaction, resulting in an increase in pulp brightness. However, too high chemical 
concentrations will cause excessive $\mathrm{H}_{2} \mathrm{O}_{2}$ decomposition and increase $\mathrm{H}_{2} \mathrm{O}_{2}$ consumption, leading to a low bleaching efficiency $[9,10]$.

Much research has focused on the improvement of $\mathrm{H}_{2} \mathrm{O}_{2}$ bleaching efficiency by controlling chemical concentrations in the bleaching system. To overcome the inadequate alkaline concentration in the later stage of conventional bleaching, Lachenal [11] suggested that $\mathrm{NaOH}$ may be charged in the middle period of the bleaching reaction for reactivating the residual $\mathrm{H}_{2} \mathrm{O}_{2}$ to improve brightness. Wang [12] charged $\mathrm{NaOH}$ in the $\mathrm{H}_{2} \mathrm{O}_{2}$ bleaching system after a half of total reaction time. The results showed that the secondary addition of alkali had a positive effect on the bleaching efficiency. Zhao [13] explored the $\mathrm{NaOH}$ step-addition process in the $\mathrm{H}_{2} \mathrm{O}_{2}$ bleaching of poplar CTMP. The results indicated that the $\mathrm{pH}$ change tended to more reasonable during the bleaching process, which kept the main bleaching reaction smooth and inhibited the undesired $\mathrm{H}_{2} \mathrm{O}_{2}$ decomposition.

The two-stage peroxide bleaching, which is commonly a combination of medium- and highconsistency bleaching, can relatively balance the chemical concentrations in a bleaching system. Compared to the single-stage high-consistency (HC) bleaching, about 10-20\% of bleaching chemicals can be saved in the two-stage bleaching system [14]. However, Thomas [15] stated that a two- or even three-stage bleaching process was necessarily costlier and more complex to operate than a single-stage bleaching system, which implied that a multiple-stage peroxide bleaching process may be unreasonable.

Displacement bleaching was an effective approach to improve the $\mathrm{H}_{2} \mathrm{O}_{2}$ bleaching efficiency [5,9]. In the displacement bleaching, high amounts of alkaline hydrogen peroxide solution were passed through a pulp bed until the desired chemical concentrations in the pulp bed were reached. Those chemical concentrations were relatively low and stable compared to that in conventional HCbleaching, which contribute to inhibition of $\mathrm{H}_{2} \mathrm{O}_{2}$ decomposition [16]. Zhao [8] studied the displacement bleaching for poplar CTMP. The results indicated that the bleaching efficiency reached 8.03 when the brightness gain was $32.0 \%$ ISO (from 44.7\%ISO to 76.7\%ISO). It is noted that the displacement bleaching technology for chemi-mechanical pulp has not been applied in the practice yet. It may be one of important reasons that the bleaching equipment is complex and costly.

Although the above-mentioned approaches had improved the $\mathrm{H}_{2} \mathrm{O}_{2}$ bleaching efficiency to a varying extent, there is considerably large room for further improvement [13]. Continuous Batch Cooking (CBC) is an improved batch cooking technology [17,18]. The main characteristic of the $\mathrm{CBC}$ is that the chemical concentration is relatively stable by continuously supplying the fresh cooking chemicals in the cooking. Can we apply this idea to the improvement in the $\mathrm{H}_{2} \mathrm{O}_{2}$ bleaching efficiency of high-yield pulps? In this paper, the authors presented a novel method, namely the 
Continuous Chemical Supplement (CCS), for peroxide bleaching of high-yield pulps. Using this method, supplementary chemical solutions were continuously pumped into the bleaching system by peristaltic pumps during bleaching, aiming at maintaining a constant chemical concentration to improve the $\mathrm{H}_{2} \mathrm{O}_{2}$ bleaching efficiency. The brightness of pulp, bleaching efficiency and chemical concentrations in bleaching system were characterized.

\section{Experimental}

\section{Materials}

The unbleached poplar CTMP was supplied by Shandong Taiding New Material Co. Ltd (Shandong, China). It was collected from the blowline of the high-consistency refiner before the bleaching stage, then washed using de-ionized water, and then dewatered. The prepared pulp was stored in a cold room at about $4{ }^{\circ} \mathrm{C}$.

\section{Chemicals}

The following chemicals are commercially available. $\mathrm{Na}_{2} \mathrm{SiO}_{3}\left(41^{\circ} \mathrm{Bé}\right)$ was from China PQ (Tianjin) Silicates Technology Co. Ltd. Stabilizer, an organophosphonate compounds, was provided by Chunjiang Chemicals Co. Ltd (Changzhou, China). Magnesium hydroxide was from Haililong Magnesium Technology Co. Ltd (Weifang, China). Diethylenetriaminepentaacetic acid (DTPA) was from Akzo Nobel N.V (Amsterdam, Netherlands).

\section{Experimental apparatus for the CCS bleaching}

The CCS bleaching experiments were done in a set-up, which process scheme was shown in Fig. 1. The reactor is a beaker (2) with a volume of $2000 \mathrm{~mL}$ that was placed in thermostatic bath (1) ( \pm $0.1{ }^{\circ} \mathrm{C}$ precision, Model THCY-18Q, Ningbo Tianheng Instrument Factory, China). A stirrer (3) with a rotating speed of 50-2000 r/min (IKA-WERKT Company, Germany) was used to mix pulp

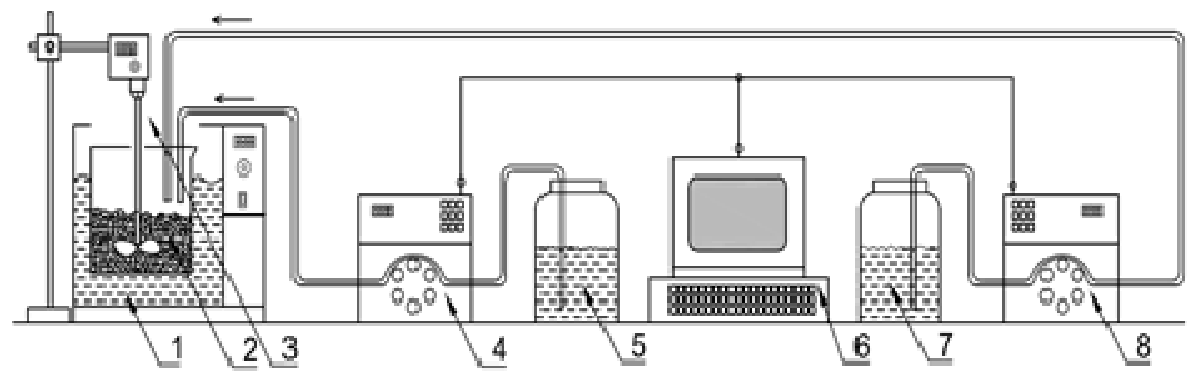

Fig. 1 Apparatus used in IC bleaching

Note: 1- thermostatic bath, 2- beaker, 3- stirrer, 4- peristaltic pump for $\mathrm{H}_{2} \mathrm{O}_{2}$, 5- plastic container for $\mathrm{H}_{2} \mathrm{O}_{2}$,

6-computer, 7- plastic container for $\mathrm{NaOH}, 8$ - peristaltic pump for $\mathrm{NaOH}$

with chemicals. Two 500-mL plastic containers (5 and 7) were filled with supplementary solutions, respectively. Two peristaltic pumps (4 and 8) (Model BT100S-YZ15, with a flow rate of 22 $\mu \mathrm{L} /$ r.min, Baoding Lead Fluid Technology Co., Ltd, Hebei, China) pumped the supplementary 
solutions from the two plastic containers into the beaker reactor, respectively. In order to reach constant chemical concentrations in bleaching system, the rotation speeds of the two pumps were controlled by a computer (6) to timely alter the charge of supplementary chemicals.

\section{Procedure of the CCS bleaching}

At the beginning of each CCS bleaching experiment, a group of estimated rotation speeds for peristaltic pumps were entered into a software program that was installed on the computer. A certain amount of water was placed in the beaker reactor and heated to $75{ }^{\circ} \mathrm{C}$ in the thermostatic bath. After that, a 40.00-g unbleached poplar CTMP was placed in the reactor, and then the stirrer was started to rotate at the speed of $200 \mathrm{rpm}$. When the consistent pulp suspension was formed and the temperature was reached, the following bleaching chemicals were sequentially mixed with the unbleached pulp: DTPA, $\mathrm{Mg}(\mathrm{OH})_{2}$, stabilizer, $\mathrm{Na}_{2} \mathrm{SiO}_{3}, \mathrm{NaOH}$ and $\mathrm{H}_{2} \mathrm{O}_{2}$. A small amount of water was lastly added into the bleaching system for the set pulp consistency of $4 \%$. After that the bleaching reaction began. Immediately, the two peristaltic pumps were started and the supplementary solutions of $\mathrm{H}_{2} \mathrm{O}_{2}$ and $\mathrm{NaOH}$ were respectively added into the beaker reactor for 50 min. During bleaching, the concentrations of peroxide and total alkali in bleaching system was monitored by withdrawing samples at specified times. If the measured chemical concentration differs from the required one, the rotation speed of peristaltic pump should be varied to calibrate it. Such operation may be repeated for many times until the chemical concentrations in bleaching system remained constant $( \pm 0.05 \mathrm{~g} / \mathrm{L}$ acceptable deviation). Thus, the group of calibrated rotation speeds were entered into a software program and applied in further experiments.

A CCS bleaching operation was carried out. After the retention of $50 \mathrm{~min}$, the pulp in the reactor was completely transferred into a cloth bag, and then thickened to an about $30 \%$ consistency. The consistency and weight of the thickened wet pulp were determined to calculate the pulp yield. The obtained filtrate was cooled down to room temperature and then determined the residual peroxide concentration.

The thickened wet pulp weight of $60.00 \mathrm{~g}$ was immediately sealed in a plastic bag, and then placed in the other thermostatic water bath where the temperature had risen to $90{ }^{\circ} \mathrm{C}$. The bleaching reaction continued for $70 \mathrm{~min}$. After the required retention time, the bleached pulp was cooled down and diluted exactly to $5.0 \%$ pulp consistency. The diluted pulp was then thickened again to an about $30 \%$ consistency. The obtained filtrate was used to measure and calculate residual peroxide concentration. The thickened pulp was washed to neutral $\mathrm{pH}$ and used to determine the final brightness. The bleaching efficiency is calculated. 


\section{Experimental design for the CCS bleaching}

The initial concentrations of $\mathrm{H}_{2} \mathrm{O}_{2}, \mathrm{NaOH}$, and $\mathrm{Na}_{2} \mathrm{SiO}_{3}$ in bleaching system commonly are three important factors to influence properties of bleached pulp [19-21], which were investigated in the CCS bleaching (Table 1). The following experimental conditions were constant for the all experimental runs: $0.2 \% \mathrm{Mg}(\mathrm{OH})_{2}, 0.3 \%$ stabilizer, $0.2 \%$ DTPA, $4 \%$ pulp consistency, $75{ }^{\circ} \mathrm{C}$, and maintaining constant concentrations of $\mathrm{H}_{2} \mathrm{O}_{2}$ and total alkali for $50 \mathrm{~min}$. After this time elapsed, the pulp was thickened to about $30 \%$ consistency, and continuously bleached at $90^{\circ} \mathrm{C}$ for $70 \mathrm{~min}$.

\section{Procedures of HC bleaching}

In order to compare the effectiveness of the CCS bleaching, the same pulp was bleached in a conventional $\mathrm{HC}$ bleaching process. The experimental conditions of $\mathrm{HC}$ bleaching, which were typically found in mills, were as follow: $5.5 \% \mathrm{H}_{2} \mathrm{O}_{2}, 2.75 \% \mathrm{NaOH}, 3 \% \mathrm{Na}_{2} \mathrm{SiO}_{3}, 0.2 \% \mathrm{DTPA}, 25 \%$ pulp consistency. All bleaching chemicals and additional water were thoroughly mixed with unbleached pulp weight of $40 \mathrm{~g}$. The mixture was sealed in a plastic bag, then immediately placed in a thermostatic water bath, at $90{ }^{\circ} \mathrm{C}$ for $120 \mathrm{~min}$. After the required retention time, the pulp was cooled down and diluted exactly to $5.0 \%$ pulp consistency. The residual peroxide in bleached pulp was determined, and the amount of $\mathrm{H}_{2} \mathrm{O}_{2}$ consumption was calculated. The brightness of the bleached pulp was determined and the bleaching efficiency was obtained.

\section{Measurements}

Pulp brightness was determined using an L\&W Elrepho Autoline 300 spectrophotometer according to ISO standard method 2470-1:2009. Brightness pads (approximately $200 \mathrm{~g} / \mathrm{m}^{2}$ ) for the brightness test were prepared according to ISO standard method 3688:1999 (E). The automatic potentiometric titrator (AT-510, Kyoto Electronics Mfg. Co. Ltd, Japan) was used to determine the concentrations of $\mathrm{H}_{2} \mathrm{O}_{2}, \mathrm{NaOH}$, and total alkali [22].

\section{Results and discussion}

\section{The relationship between pulp brightness and effectiveness in the CCS bleaching}

As described in the introduction, the status of the chemical concentration of the hydrogen peroxide bleaching system affects the bleaching efficiency. The constant concentrations of $\mathrm{H}_{2} \mathrm{O}_{2}$ and total alkali in the bleaching system were maintained at different levels, and the brightness and bleaching efficiency were significantly different (Table 1). The maximum values of the bleaching efficiency were 18.00, and the simultaneous brightness was $74.98 \%$ ISO (No. 3 experimental run). The highest value of brightness was $77.86 \%$ ISO, and the obtained bleaching efficiency was 10.83 (No. 13 experimental run). The experimental element with a high brightness usually had a low bleaching efficiency. These results indicated that the maximum brightness and bleaching efficiency might not 
be able to be obtained at the same time under the same experimental conditions, although this is the ideal goal for bleaching experiments.

Table1. The effects of chemical concentrations on bright and bleaching efficiency

\begin{tabular}{|c|c|c|c|c|c|}
\hline Run & $\begin{array}{l}\mathrm{H}_{2} \mathrm{O}_{2} \\
(\mathrm{~g} / \mathrm{L})\end{array}$ & $\begin{array}{c}\mathrm{NaOH} \\
(\mathrm{g} / \mathrm{L})\end{array}$ & $\begin{array}{c}\mathrm{Na}_{2} \mathrm{SiO}_{3} \\
(\mathrm{~g} / \mathrm{L})\end{array}$ & $\begin{array}{c}\text { ISO brightness } \\
(\%)\end{array}$ & bleaching efficiency \\
\hline 1 & 1.78 & 1.02 & 2.50 & 72.76 & 14.62 \\
\hline 2 & 1.78 & 0.51 & 3.75 & 72.00 & 16.65 \\
\hline 3 & 3.57 & 0.51 & 2.50 & 74.98 & 18.00 \\
\hline 4 & 3.57 & 1.02 & 3.75 & 75.84 & 13.84 \\
\hline 5 & 3.57 & 1.53 & 2.50 & 75.80 & 10.18 \\
\hline 6 & 1.78 & 1.02 & 5.00 & 73.04 & 15.05 \\
\hline 7 & 1.78 & 1.53 & 3.75 & 72.60 & 12.93 \\
\hline 8 & 5.35 & 0.51 & 3.75 & 76.36 & 13.84 \\
\hline 9 & 3.57 & 0.51 & 5.00 & 74.32 & 13.69 \\
\hline 10 & 3.57 & 1.53 & 5.00 & 75.98 & 13.63 \\
\hline 11 & 3.57 & 1.02 & 3.75 & 75.16 & 13.63 \\
\hline 12 & 5.35 & 1.02 & 5.00 & 77.29 & 12.96 \\
\hline 13 & 5.35 & 1.53 & 3.75 & 77.86 & 10.83 \\
\hline 14 & 5.35 & 1.02 & 2.50 & 77.53 & 13.02 \\
\hline 15 & 3.57 & 1.02 & 3.75 & 75.52 & 12.91 \\
\hline 16 & 3.57 & 1.02 & 3.75 & 75.92 & 13.72 \\
\hline 17 & 3.57 & 1.02 & 3.75 & 75.46 & 12.25 \\
\hline
\end{tabular}

\section{The effects of chemical concentrations on bleaching efficiency}

The concentrations of hydrogen peroxide and total alkali in bleaching system were monitored and compared (Figs. 2 and 3) in different bleaching process. The initial concentrations of total alkali and hydrogen peroxide in CCS bleaching were $4.5 \mathrm{~g} / \mathrm{L}$ and $0.79 \mathrm{~g} / \mathrm{L}$, which were kept unchanged for $50 \mathrm{~min}$. The initial concentrations of hydrogen peroxide and total alkali in $\mathrm{HC}$ bleaching were $19.5 \mathrm{~g} / \mathrm{L}$ and $10.3 \mathrm{~g} / \mathrm{L}$, which reduced sharply in the early bleaching stage and enter into a slow reduction period after about $20 \mathrm{~min}$. The hydrogen peroxide concentration in CCS bleaching was relatively low, while its total alkali concentration was low in the initial stage but got higher than those in HC bleaching after 5 and 15 min, successively. The bleaching efficiency in the CCS bleaching was 16.5, which was significantly higher than 7.4 in HC bleaching (Table 2). When the brightness was compared, the brightness obtained in the CCS bleaching was $75.83 \%$ ISO, which was highly close to $76.42 \%$ ISO in HC bleaching. This result indicated that the CCS bleaching was an effective beaching method to improve bleaching efficiency. The concentrations of total alkali and hydrogen peroxide in the CCS bleaching were relatively low in the early bleaching stage, which was beneficial to the inhibition of hydrogen peroxide decomposition. Meanwhile, the total alkali 
concentration were higher in the mid-late stage, which promoted the generation of hydrogen peroxide anions $\left(\mathrm{HOO}^{-}\right)$. Therefore, the comparably high bleaching efficiency reached in the CCS bleaching

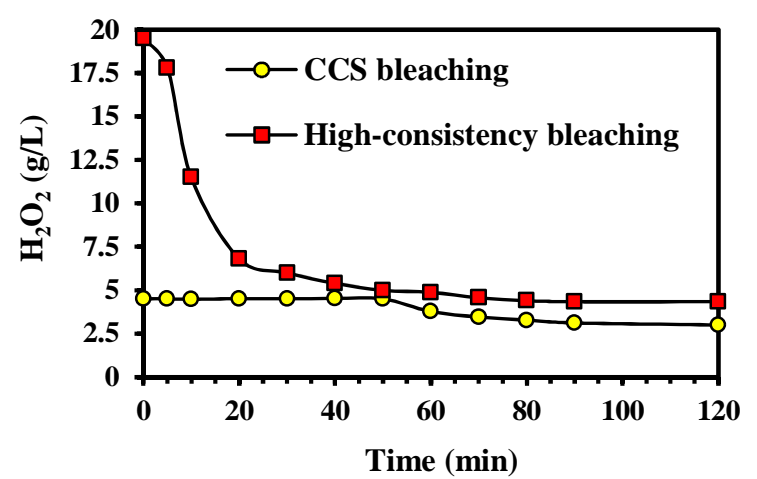

Fig. $2 \mathrm{H}_{2} \mathrm{O}_{2}$ concentrations in bleaching

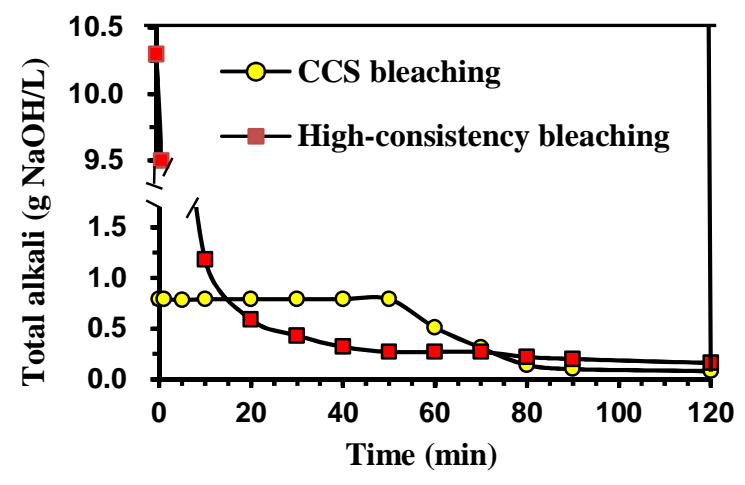

Fig. 3 total alkali concentrations in bleaching

\section{The effect of temperature on pulp brightness and effectiveness in CCS bleaching}

The developments of bleached pulp at different temperature level were monitored in the CCS process. The rightness increased rapidly at the early stage of bleaching period. A majority of bleaching reaction have been finished in this period. A similar tendency in HC bleaching has also been suggested by previous studies $[15,10]$. When temperature increased from $65{ }^{\circ} \mathrm{C}$ to $95{ }^{\circ} \mathrm{C}$, the brightness increased but the bleaching efficiency decreased from 17.8 to 6.8 (Fig. 4). This result indicated that an increase in temperature can accelerate bleaching reaction resulting high brightness. However, high temperature can also accelerate the undesired $\mathrm{H}_{2} \mathrm{O}_{2}$ decomposition resulting low bleaching efficiency $[19,20,23]$.

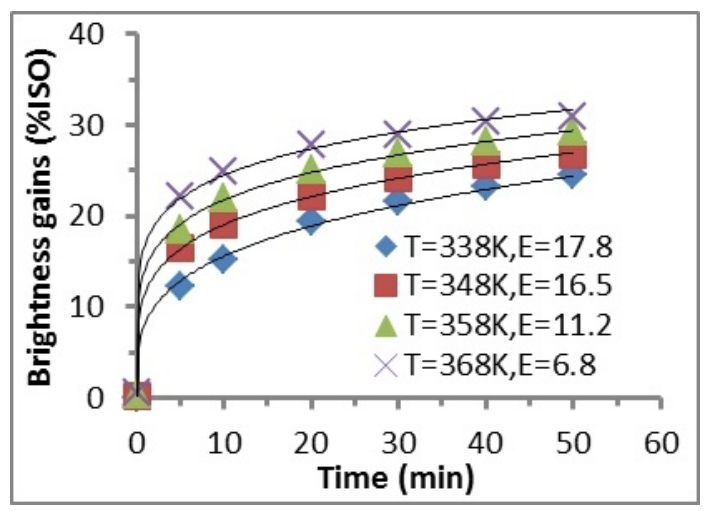

Fig. 4 the bleaching efficiency and in different bleaching system

The previous studies have suggested that balancing chemical concentration has positive effects on peroxide bleaching efficiency. However, those balanced concentrations were relatively stable, but completely constant. In addition, most of studies have focused only on total alkali concentration. Few researchers have attempted to balance $\mathrm{H}_{2} \mathrm{O}_{2}$ concentration in bleaching system. Our results suggested that maintaining the completely constant concentrations for a long time tends to obtain higher bleaching efficiency. The undesired decomposition of $\mathrm{H}_{2} \mathrm{O}_{2}$ was inhibited due to 
the low initial chemical concentrations at the beginning of bleaching reaction, and the brightness was improved due to adequate chemical concentrations at the end of the bleaching reaction. This method resolved essentially the problem that the chemical concentrations are too high at the early stage, but too low at the end stage in conventional bleaching. The bleaching efficiency, therefore, was improved significantly.

Most notably, this is the first study to our knowledge to maintaining constant chemical concentration by pumping continuously chemical solution into a bleaching system for peroxide bleaching of CTMP. Our findings were promising and should be explored further. Future work should focus on maintaining a constant stabilizer concentration. The bleaching temperature, time, and additives species also should be further optimized for the further improvement of bleaching efficiency.

\section{Conclusions}

In this study, we present a new method of improving peroxide bleaching efficiency for poplar CTMP. This method used two peristaltic pumps to continuously pump $\mathrm{H}_{2} \mathrm{O}_{2}$ and $\mathrm{NaOH}$ solutions into the bleaching system to maintain the constant concentrations of $\mathrm{H}_{2} \mathrm{O}_{2}$ and total alkali. It solved the problem that the chemical concentrations are too high at the early stage, but too low at the end stage in conventional peroxide bleaching. Our results suggested the CCS bleaching was an effective method to improve bleaching efficiency. This method could be applied to industrial production to reduce dramatically chemical cost. Further studies in this field should be encouraged.

\section{References}

[1] Pan G: Pulp \& Paper Canada Vol. 102-11, (2001), P. 41-45

[2] Zhou Y, Zhang D, Li G: China Pulp \& Paper Vol. 24-5, (2005), P. 51-60

[3] Wang B: China Pulp \& Paper (z1): (2006), P. 33-38

[4] Hatam A, Pourtahmasi K, Resalati H, Lohrasebi AH: Wood SciTechnol Vol. 42, (2008), P. 353367

[5] Li X: Studies on hydrogen peroxide displacement bleaching processes and mechanisms of Eglobulus CTMP. Dissertation, South China University of Technology (2000)

[6] Xu E: Journal of Pulp and Paper Science Vol. 28-11, (2002), P. 379-383

[7] Zhao Q, Pu J, Mao S, Qi G: BioResources Vol. 5-1, (2010),P. 276-290

[8] Zhao Q: Research on reinforced hydrogen peroxide bleaching of poplar CMP and bleaching mechanism. Dissertation, Beijing Forestry University (2009)

[9] Zeinaly F, Shakhes J, Firozabdi MD, Shakeri A: BioResources Vol. 4-4 (2009), P. 1409-1416 
[10] Shen K: High-brigthness bleaching of China fir CTMP pulp and its mechanism. Dissertation, Chinese Academy of Forestry (2008)

[11] Lachenal D, Dubreuil M, Bourson L: TAPPI Vol. 73-10 (1990), P. 195-199

[12] Wang R: Studies on anaphase activation of peroxide bleaching of pulp. Dissertation, Northeast Forestry University (2004)

[13] Zhao Q, Sun D, Wang Z, Pu J, Jin X, Xing M: BioResources Vol. 7-4 (2012), P. 4889-4901

[14] Suhr M, Klein G, Kourti I, Gonzalo MR, Santonja GG, Roudier S, Sancho LD: Best available techniques (BAT) reference document for the production of pulp, paper and board. European Integrated Pollution Prevention and Control Bureau. (2014) doi: 10.2791/370629

[15] Thomas HC, Winkler WF, Lee JA: Process for high temperature peroxide bleaching of pulp with cool discharge. Unite State patent NO.7297225 B2 (2007)

[16] Li Z, Ni Y, Heiningen AV: Pulp \& Paper Canada Vol. 97-9 (1996) P. 46-50

[17] Fardim P, Akademi Á: Chemical pulping part1, fiber chemistry and technology. In Papermaking science and technology 2nd edn, book 6. Finnish Paper Engineers' Association and TAPPI, Helsinki (2011)

[18] Jafari V, Sixta H, Heiningen AV: Industrial \& Engineering Chemistry Research (2014).doi: 10.1021/ie403336x

[19] Sundholm J: Mechanical pulping. In Gullichsen J, Paulapuro H (ed) Papermaking science and technology, book 5. Finnish Paper Engineers' Association and TAPPI. Helsinki (1999)

[20] Ek M, Gellerstedt G, Henriksson G: Pulping chemistry and technology. In: Pulp and paper chemistry and technology, volume 2 (2009)

[21] Fei X, Yao J, Du J, Sun C, Xiang Z, Xu C: Cellulose Vol. 22 (2015), P. 1379-1388

[22] Hietanen TM, Österberg M, Backfolk KA (2013) Effects on pulp properties of magnesium hydroxide in peroxide bleaching. BioResources 8(2):2337-2350

[23] Mota SF, Paulo JG, Zeller CAV, Ferreira LM, Carvalho MGV: Nordic Pulp and Paper Research Journal Vol. 22-1: (2007) P. 17-22 